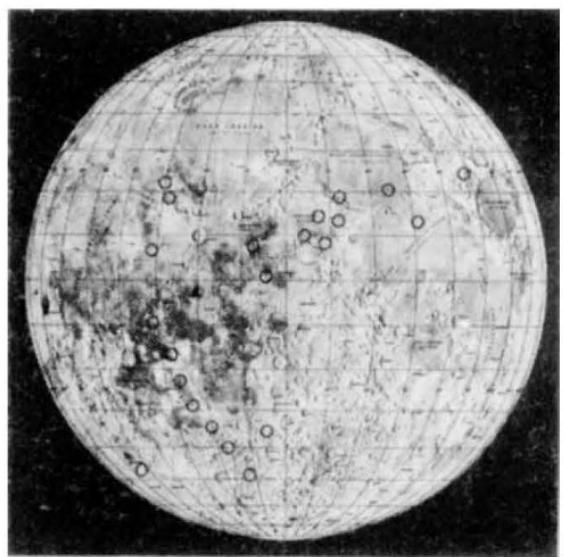

The near side of the Moon showing the epicentres of moonquakes recorded by lunar-based seismometers.

$s^{-1}$ ) in free flight above the lunar surface (Dr W. von Engelhardt, University of Tübingen). The orange soil near the Apollo 17 site was shown to consist chiefly of coloured glass. The most striking region, located in a cylinder $1 \mathrm{~m}$ in diameter and $0.3 \mathrm{~m}$ deep, showed no evidence of recent volcanic activity, but was formed nearly $4 \times 10^{9} \mathrm{yr}$ ago and has been exposed near the surface for about $3 \times 10^{7} \mathrm{yr}$.

The installation of a network of lunarbased seismometers has led to some of the most impressive results of the whole Apollo programme. The outer 20 to 30 $\mathrm{km}$ of the Moon has been shown to be broken or fractured so that the propagation of seismic energy within this layer may be regarded as a diffusion process. The seismometers have also detected the impact of many large meteorites on the lunar surface, measuring both the energy and position of the impact. The statistics of such observations are in good agreement with other (often less reliable) estimates of meteorite flux.

Internal centres of lunar seismic activity have been located principally between 800 and $1,100 \mathrm{~km}$ below the surface (lunar radius $1,738 \mathrm{~km}$ ). At the conference Dr G. Latham (University of Texas, Galveston) reported that the epicentres for such events (moonquakes) lie close to two great circles (see Figure). $\mathrm{He}$ also discussed the impact of a large meteorite near the Sea of Moscow on the lunar far side. The shear waves from this impact were completely suppressed by the central zone of the lunar interior and this may imply central temperatures as high as $1,800 \mathrm{~K}$-a value many hundreds of degrees above that inferred earlier from observations of the response of the Moon to the electromagnetic field associated with the solar wind.

The sites chosen for the Apollo landings have moved progressively from the flat dark mariai regions to lighter more rugged upland areas. The last two landings, in particular, have investigated regions with kilometre scale mountains, large boulders many metres in size and even outcrops of rock. Subsequent terrestrial analysis of these rocks has shown that very few of the samples are similar to the Apollo 11 and 12 basalts, formed in the maria by solidification of large volumes of magma or lava. Instead these upland rocks are breccias, that is composite rocks formed by the compaction of powdered or broken rock under the influence of temperature or pressure. Breccias were also returned from the maria but the upland breccias are composed of particles of comparatively primitive anorthitic rock. These breccias range from fragile low-density samples to dense rocks which have undergone heat treatment in the lunar surface layer at temperatures well above $1,000 \mathrm{~K}$ and in some cases almost sufficient to return them to molten magma. This history has been confirmed by magnetic measurements reported at the meeting which indicate cooling from temperatures above the Curie point in the presence of magnetic fields estimated in the range $10^{-4}$ to $10^{-6}$ tesla ( 1 to $10^{-2}$ oersted). Still higher fields are required by magnetometer measurements carried out on the lunar surface. A dynamo mechanism in a once liquid lunar core could possibly produce such a field, but a more attractive possibility may be an early intense solar field invoked in modern condensation and accretion models for the origin of the Solar System.

Whatever the origin of this early magnetic field its discussion at this conference has demonstrated clearly that lunar science is not simply an isolated study. The antiquity of the lunar surface on a cosmological time scale makes it ideal for experiments concerning changes in the rate of solar flare activity, the flux of meteorites and the cosmic-ray flux. Rarely regarded as intellectually respectable, erratically if lavishly supported by a few countries, lunar science has now returned to the stage where its adherents are chiefly motivated by scientific interest.

\section{GLOMAR CHALLENGER Red Sea Drilling}

from our Geomagnetism Correspondent IN view of the importance of the Red Sea as a region of apparently young seafloor spreading, the results of the visit to the area last year by the deepsea drilling vessel Glomar Challenger are awaited with some anticipation. Full details will presumably become available slowly over the coming months and years; but in the meantime Ross et al. (Science, 179, 377; 1973) have reported preliminary results and conclusions derived on board during the voyage.

Three of the six holes drilled (225 to 230) were in the vicinity of the wellknown hot brine area comprising the Atlantis II, Chain and Discovery Deeps where waters of high salinity and temperature are underlain by sediments enriched in heavy metals such as copper, lead, zinc, silver and gold. The core from site 225, which lies $16 \mathrm{~km}$ east of the Atlantis II Deep, indicated $177 \mathrm{~m}$ of late Miocene to late Pleistocene silt and ooze overlying $53 \mathrm{~m}$ of anhydrite and halite. The surface of these evaporites was found to correlate with the acoustic reflector $S$ (velocity $4.4 \mathrm{~km} \mathrm{~s}^{-1}$

\title{
Amino-acids in Lunar Samples
}

IN next Monday's Nature Physical Science (March 26) Modzeleski et al. report their analyses of seven samples brought back from the Moon by the Apollo 14 astronauts for carbon compounds and amino-acids. They used a combined vacuum pyrolysis-mass spectrometry technique for estimating gases containing carbon and hydrocarbons and ion exchange chromatography for quantifying the amino-acids. These latest results are part of an ongoing attempt to elucidate the history and manner of formation of these molecules on the Moon.

The principal gaseous products of the pyrolysis procedure were $\mathrm{CO}, \mathrm{CO}_{2}$ and $\mathrm{CH}_{4}$, and the results of pyrolysing $50 \mathrm{mg}$ portions of the lunar samples indicated that carbon was present in the gases to the extent of between 76 and 161 parts per million. The largest relative amount of $\mathrm{CO}$ and $\mathrm{CO}_{2}$ was given off by a breccia sample rather than by a sample of fines.

When the mass spectrometer was set to record compounds with a larger molecular weight, many species came to light, for example $\mathrm{C}_{2} \mathrm{H}_{5}, \mathrm{C}_{3} \mathrm{H}_{5}$ and COS. And sulphur was clearly evident as a ring above the cold trap of the pyrolysis tube. Modzeleski et al. say that their findings suggest that the sulphur was not present in elemental form nor in an organic compound but probably in a mineral in view of the high temperature needed to liberate it.

The ion exchange column used to analyse aqueous extracts of the lunar samples was capable of detecting as little as $5 \times 10^{-11} \mathrm{~mol}$ of amino-acid. As it turned out, all the amino-acids detected were present in quantities less than $8 \times 10^{-11} \mathrm{~mol}$ per gram of extract, except for glycine. Glycine, aspartic acid, glutamic acid and serine were the most abundant of the amino-acids detected. Tests on parts of the surface of one of the astronaut's gloves revealed no amino-acids, indicating that those detected did in fact come from the Moon. 